\section{Cold dark matter makes an exit}

\author{
David Lindley
}

WHEN. about two decades ago, cosmologists began to apply statistical analysis to the distribution of galaxies in the sky, one of the first results was a schism in the theoretical world. The article of faith on which this schism hinged was the existence or not of structures in the distribution of galaxies - superclusters, filaments, giant voids - on very large scales. Over the years, evidence has mounted that big structures really do exist, and the paper by Saunders et al. in this week's issue seems finally to prove the point - and in so doing, disposes of the favoured "cold dark matter' model of galaxy formation.

The statisticians discovered early on that the two-point correlation function, which measures the difference between the observed galaxy distribution and a strictly random one, diminishes smoothly for increasing galaxy separation, suggesting that whatever mechanism formed galaxies operated on small scales, but petered out on large scales, so that any large-scale structures were merely the predictable aggregates of the small-scale clustering. But several observers obstinately insisted that the filaments and voids they could see plainly in galaxy maps were more than the two-point correlation function could account for.

\section{Theoretical preference}

It was hard, however, for the disbelievers to come up with any unambiguous statistics with which to prove that bona fide large-scale structures existed. Theoretical preferences hinged on this observational debate. In 'bottom up' theories of galaxy formation, structures formed first on small scales and aggregated into larger units; in 'top down' theories, the large structures were primary, and fragmented into small pieces.

This debate now seems, in hindsight, rather pointless. As cosmologists attacked the galaxy distribution, it became apparent that the existence of a smooth correlation function on small scales does not preclude the existence of large-scale structures (this is only a fancy way of saying that an infinite number of statistical distributions can have the same mean and standard deviation but have very different higher-order moments).

Equally, evidence mounted that large structures really do exist. Recently we have had the "Great Attractor' a giant congregation of galaxies and clusters noticeable by the gravitational pull it exerts on its neighbouring galaxies, the "Great Wall', a continuous sheet of galaxies stretching across the sky and, with less certainty, periodicity in the galaxy distribution on a 100 -megaparsec scalet.
The problem is then to produce a model which can account for the small-scale structures, characterized as ever by the two-point correlation function, but also permits the possibility of large-scale structures. The disparate nature of the various large structures means that one should not hope to predict them in any specific way: it is sufficient to show that one's theory harbours some reasonable probability that such a structure might arise in the volume of the observable universe.

For a few years, the best attempt at such a comprehensive theory appeared to be the cold dark matter (CDM) model. Dark matter is the invisible stuff that dynamical studies of galaxies and clusters of galaxies indicate must be there, but which can't be seen; the fact that it is cold means that the particles which compose it (entirely hypothetical particles, it must be said) are slowly moving, a property that enables them to clump together into galaxy-sized lumps.

But now Saunders et al.' say that the CDM theory, like many of its predecessors, must be discarded. They argue that the picture of the galaxy distribution given by the Infrared Astronomy Satellite (IRAS) is, on the largest scales, in clear have. This disavowal of CDM is all the more remarkable for coming from a group of authors that includes some of the theory's long-time supporters. statement because they avoid arguments about whether CDM or any other theory can explain such particular observational features as the Great Attractor or the Great Wall. Both the Greats are clearly unique aggregations, and although their existence in CDM or any other theory may be a little unlikely, cosmologists could carry on believing their favourite theory provided they were willing to accept a couple of low-probability items in their version of the heavens. But the IRAS survey allows the well-studied correlation-function technique to be applied on the scale of the whole sky. Infrared observations avoid most of the extinction problems which limit the sky-coverage and uniformity of a comparable optical survey, and by producing a survey that covers almost the whole sky to uniform depth, IRAS generates a catalogue of galaxies from which one can obtain, for the first time, a believable correlation function over a very large range of scales.

The CDM model slips up because it does not get the galaxy distribution right on scales of about 20 megaparsecs and above: the real sky has more nonrandomness than CDM can provide. The reason CDM fails is essentially that it was contradiction with what CDM would

Saunders et al. can make this definitive designed to explain structure at the level of galaxies and small clusters of galaxies. In the early days of dark-matter cosmologies, the idea that neutrinos might have a cosmologically interesting mass was in vogue, and although neutrinos may indeed have a mass that will interest cosmologists ${ }^{5.6}$, the fact that they must be moving relativistically makes it almost impossible for them to aggregate in gravitationally bound associations on the scale of individual galaxies. CDM, by contrast, works on the galactic scale. Large scales were never imagined to be its forte, but only with the work of Saunders et al. has quantitative cvidence of this inadequacy come to light.

\section{Cosmological constant}

Where does this leave galaxy-formation theory, and cosmology in general? Some of Saunders's colleagues have suggested that CDM can be saved, at least in modified form, if a non-zero cosmological constant is resurrected ${ }^{7.8}$; a cosmological constant, amounting to a vacuum energy density, modifies cosmic dynamics on the large scale, but leaves the small scale alone, compensating for CDM's weakness. Alternatively, perhaps neutrinos really do have a cosmologically significant mass $^{5.6}$, so that one can imagine the dark matter being mostly CDM for individual galaxies and mostly neutrinos for the giant structures.

If cosmologists are forced to start throwing around several kinds of dark matter, along with a cosmological constant, scepticism is bound to arise: a sufficiently complicated model can always replicate, in the manner of Ptolemy's epicycles, a limited set of observations.

Ptolemy's Solar System was satisfactorily replaced by one dictated by a single underlying principle, the inverse-square law of gravitation. But do we have any right to expect so messy a subject as galaxy formation to be likewise guided by a single idea? If particle physicists prove tomorrow that the neutrino has a mass of 4.73 electronvolts, and that a species of previously unknown particle provides 0.62 of a critical cosmic density in the form of cold dark matter, cosmologists could confidently set themselves to constructing messy theories with these ingredients. But if cosmologists declared, in the absence of independent evidence, that only with these same ingredients could they explain the distribution of galaxies, would anyone believe them?

\section{David Lindley is Associate Editor of Nature.}

1. Saunders, W. et al. Nature 349, 32-38 (1991) 2. Lynden-Bell, D. et al. Astrophys. J. 326, 19-49 (1988) 3. Geller. M. \& Huchra, J. Science 246, $897-903$ (1990) 4. Broadhurst, T.J. et al. Nature 343, 726-728 (1990) Sciama, D.W. Nature 348, 617-618 (1990).

5. Sciama, D.W. Nature 348, 617-618
6. Maddox, J. Nature 348, $579(1990)$.

7. Efstathiou, G., Sutherland, W.S. \& Maddox, S.J. Nature 348, 705-707 (1990).

8. Bertschinger, E. Nature 348, 675-676 (1990) 